\title{
Bioelectroanalysis with nanoelectrode ensembles and arrays
}

\section{Michael Ongaro \& Paolo Ugo}

\section{Analytical and Bioanalytical Chemistry}

ISSN 1618-2642

Volume 405

Number 11

Anal Bioanal Chem (2013)

405:3715-3729

DOI 10.1007/s00216-012-6552-z
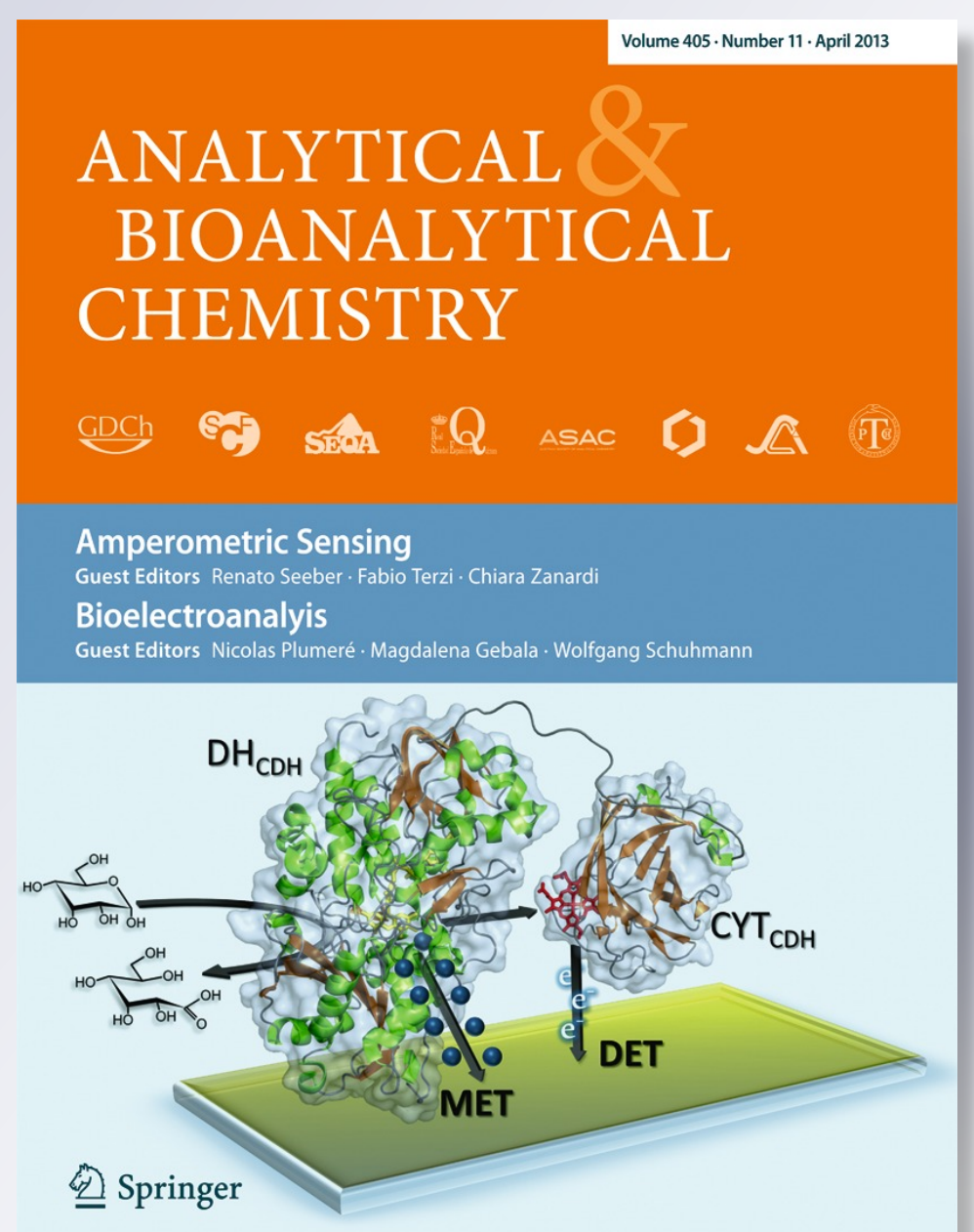

\section{望 Springer}


Your article is protected by copyright and all rights are held exclusively by SpringerVerlag Berlin Heidelberg. This e-offprint is for personal use only and shall not be selfarchived in electronic repositories. If you wish to self-archive your work, please use the accepted author's version for posting to your own website or your institution's repository. You may further deposit the accepted author's version on a funder's repository at a funder's request, provided it is not made publicly available until 12 months after publication. 


\title{
Bioelectroanalysis with nanoelectrode ensembles and arrays
}

\author{
Michael Ongaro • Paolo Ugo
}

Received: 14 September 2012 /Revised: 31 October 2012 / Accepted: 5 November 2012 /Published online: 28 November 2012

(C) Springer-Verlag Berlin Heidelberg 2012

\begin{abstract}
This review deals with recent advances in bioelectroanalytical applications of nanostructured electrodes, in particular nanoelectrode ensembles (NEEs) and arrays (NEAs). First, nanofabrication techniques, principles of function, and specific advantages and limits of NEEs and NEAs are critically discussed. In the second part, some recent examples of bioelectroanalytical applications are presented. These include use of nanoelectrode arrays and/or ensembles for direct electrochemical analysis of pharmacologically active organic compounds or redox proteins, and the development of functionalized nanoelectrode systems and their use as catalytic or affinity electrochemical biosensors.
\end{abstract}

Keywords Nanoelectrode $\cdot$ Ensemble $\cdot$ Array .

Voltammetry $\cdot$ Biosensor $\cdot$ Mediated electrochemistry

\section{Introduction}

In the last decade there has been growing interest in the development of innovative electrochemical sensors and devices for bioanalytical purposes. The final applications include biomedical diagnostics [1-3], environmental [4] and food control [5, $6]$, and safety and biohazard assessment $[7,8]$. In practice, unique characteristics distinguish (bio)electrochemical sensors from classical instrumental methods, for example spectroscopy, chromatography, and mass spectrometry; these include low cost, miniaturizability, ease of use, no interference from

Published in the topical collection Bioelectroanalysis with guest editors Nicolas Plumeré, Magdalena Gebala, and Wolfgang Schuhmann

M. Ongaro $\cdot$ P. Ugo $(\bowtie)$

Department of Molecular Sciences and Nanosystems,

University Ca' Foscari of Venice, S. Marta 2137,

30123, Venice, Italy

e-mail: ugo@unive.it coloured or turbid samples, and applicability to raw samples for "in situ" and decentralized. However, some problems and limits must still be overcome. One crucial aspect is optimization of the surface modification procedure to maximize biorecognition capabilities and reduce sensitivity to non-specific adsorption and fouling. In principle, use of sensor surfaces with appropriate nanostructure can contribute to solving some of these problems, for example by increasing the specific area available for immobilization of large amounts of the biomolecules involved in the recognition while, at the same time, keeping the overall size of the sensor very small $[9,10]$. Moreover, by separating biorecognition and transduction on the nanoscale it is possible to engineer the sensor surface so that one can protect, by use of self-assembled monolayers (SAMs) of thiols, the nanoelectrodes from undesired nonspecific adsorption yet confine biorecognition to the proximity of (but not on) the nanoelectrode [11, 12]. Use of an array of nanostructured electrodes also enables extreme miniaturization of the sensor, keeping the overall size to dimensions 1-2 orders of magnitude lower than with micrometre-sized electrodes [13]. Taking this approach to the extreme, the possibility of developing multiplexed arrays is particularly attractive $[14,15]$. For instance, Zoski et al. [14] built and tested complex arrays composed of groups of nanoelectrode ensembles, each group being individually addressable via a separate current collector.

In the following text we will discuss some relevant examples of the state of the art of preparation of ensembles and arrays of nanoelectrodes (NEEs and NEAs respectively), and models and theories explaining their electrochemical behaviour, before discussing some significant applications in bioanalysis.

Templated ensembles of nanoelectrodes

Nanoelectrode ensembles are useful electroanalytical tools which are applied in many fields ranging from sensors to 
electronics, from energy storage to magnetic materials [16]. The first template synthesis of NEEs for electrochemical use was described by Menon and Martin [17] who deposited gold nanofibres with a diameter as small as $10 \mathrm{~nm}$ within the pores of track-etched polycarbonate (PC) membranes by a chemical (electroless) method and obtained a random ensemble of metal nanodisc electrodes surrounded by the insulating polymer. All the nanoelectrodes were interconnected, so they all experienced the same electrochemical potential. A schematic diagram of the structure of an NEE is shown in Fig. 1.

Membrane-templated synthesis is based on the idea that the pores of a host material can be used as a template to direct the growth of new materials. Historically, template synthesis in track-etched materials was introduced by Possin [18] and Williams and Giordano [19], who prepared different metallic wires with diameters as small as $10 \mathrm{~nm}$ within the pores of etched nuclear damage tracks in mica. This method was designed to image the shape of the pores rather than to obtain a functional composite with electrochemical sensing capabilities, as achieved later by Menon and Martin [17]. A variety of examples of membrane templated electrochemical deposition of nanowires of semiconductors [20], metals (e.g. Ni and Co) [21], oxides, and conducting polymers [16] have subsequently appeared in the literature.

In the template synthesis of nanoelectrodes, each pore of the membrane is filled with a metal nanowire or nanofibre. Growth of the metal fibres can be achieved by use of both electrochemical $[21,22]$ or electroless $[17,23,24]$ methods of deposition.

In both methods of deposition, the pore density of the template determines the number of metal nanoelectrode elements on the NEE surface and, correspondingly, the average distance between them, whereas the diameter of

a

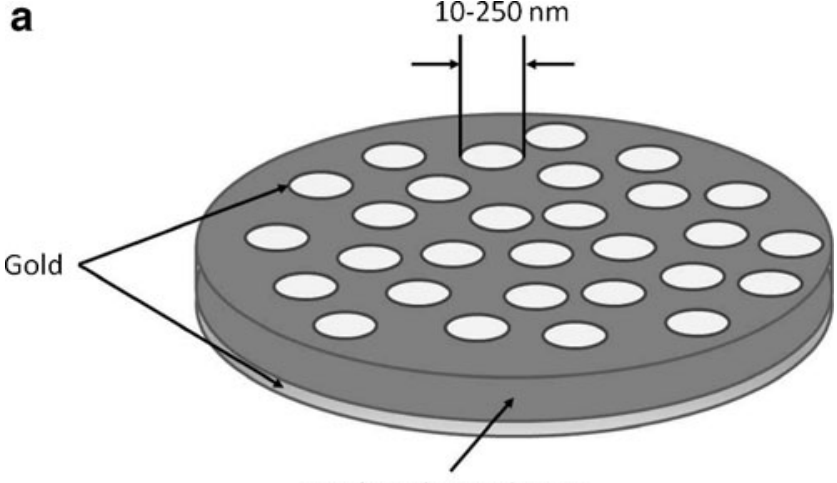

b

Track-etch membrane

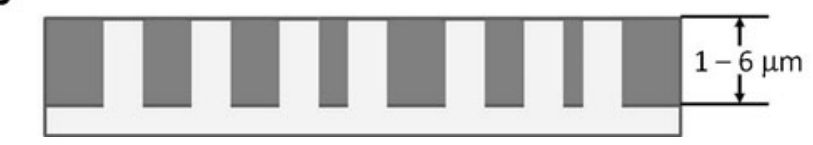

Fig. 1 Schematic diagram of a nanoelectrode ensemble in a template membrane: (a) overall view; (b) lateral section the pores in the template determines the diameter of the individual nanoelectrodes. Track-etched membranes with pore diameters ranging from $10 \mathrm{~nm}$ to $10 \mu \mathrm{m}$ are commercially available.

Template electrochemical deposition of metals

Electrochemical deposition inside the pores of a nanoporous membrane requires that one side of the membrane be made conductive. This can be achieved by plasma or vacuum deposition of a thin (typically 100-200 nm) layer of metal on one side of the membrane. The metal layer can be the same or different from the metal which will be electrodeposited inside the pores and the membrane should be sufficiently robust to tolerate this kind of treatment. As an alternative, it is possible to place the membrane directly in contact with a solid electrode. Figure 2 shows the interesting cell setup recently proposed by Gambirasi et al. [25], in which the membrane is placed between a solid electrode and a sponge drenched in the electrolyte; the pressure of the electrode on the sponge keeps the membrane fixed tightly to the electrode for the deposition time. In electrochemical template deposition, the coated film is placed in an electrochemical cell, and acts as the cathode whereas the counterelectrode is the anode.

Deposition can be performed under potentiostatic or galvanostatic conditions. In the former, it is possible to monitor the time course of the deposition and the progressive filling of the pores by analysing the time transient current. As

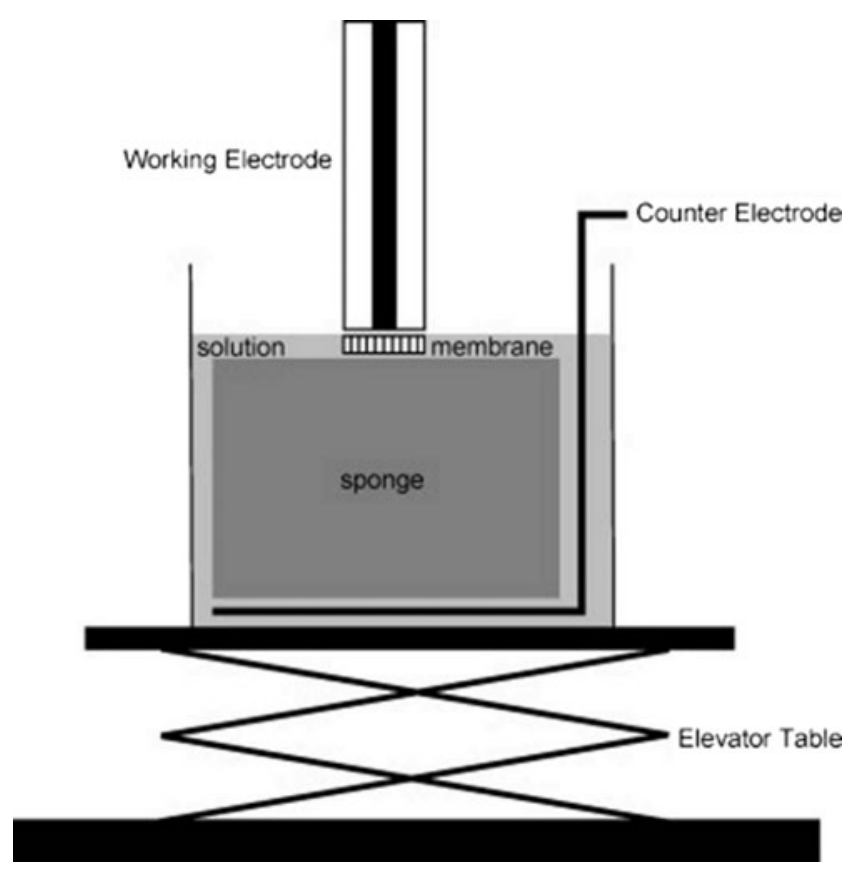

Fig. 2 Schematic representation of the cell setup. On raising the elevator the membrane lying over the sponge, soaked with the electrolyte, is pressed on the surface of the electrode (reprinted, with permission, from Ref. [25]) 
shown in Fig. 3, the deposition curve can be divided in three parts [21, 26, 27] (I-III in Fig. 3) associated with the three steps of deposition sketched below. Immediately after closing the circuit (phase I) an intense peak then rapid decay of the current is observed, because of depletion of metal ions after the rapid initial deposition and the increased resistance inside the pores of the membrane. Subsequently, the current slowly decreases, reaching a plateau (phase II) which corresponds to progressive filling of the pores. At the beginning of phase III, the current increases again because of the increase of the electrode area caused by growth of the metal outside the pores. In this phase it is possible to observe caps on the tips of the nanowires with a typical mushroom shape [21]. Finally, the overgrown caps merge together producing an almost flat surface; this leads to a second plateau in the current transient. If the objective is preparation of ensembles of nanodisc electrodes, it is essential to stop the electrodeposition at the end of stage II, i.e. before the "mushroom caps" start to grow.

Because the process is based on progressive growth and filling of the pores from the bottom metallic layer toward the open end of the pores, final products are nanowires and not hollow structures (e.g. nanotubes).

Electrodeposition of metals has been used to obtain nanowires not only of gold, but also of other materials, for example, other metals $(\mathrm{Co}[21,28,29] \mathrm{Ni}[21,26,30] \mathrm{Cu}$ [21, 26], Pt and Pd [31]), alloys (NiFe [29], FeSiB [30]), or salts $\left(\mathrm{Bi}_{2} \mathrm{Te}_{3}[32], \mathrm{CdS}[20]\right)$.

\section{Template electroless deposition}

Electroless deposition involves chemical reduction of a metal salt from solution to metal on a surface. Non-catalytic surfaces, for example insulating polymers, must be activated (made catalytic) before the electroless deposition. Usually, this is achieved by generating metal nuclei on the surface of the non-catalytic material. By this way, the metal ion is

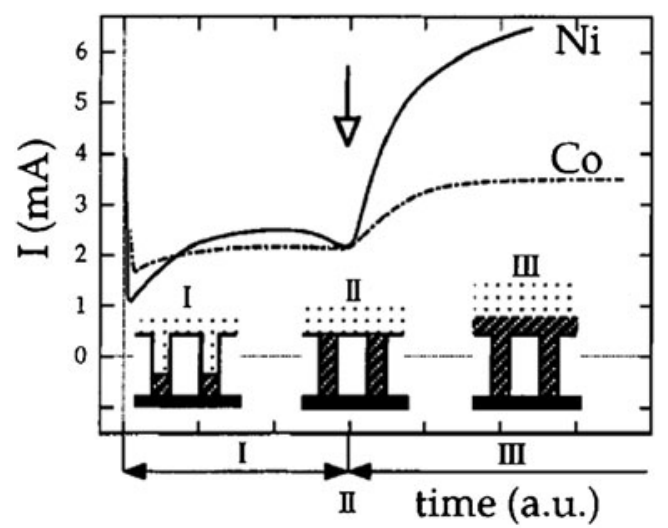

Fig. 3 Time transient current for electrochemical deposition using a track-etch membrane as templating material (reprinted, with permission, from Ref. [21]) preferentially reduced at the sensitized surface so that only this surface is finally plated with the desired metal [33].

The principles of electroless deposition on nanoporous membranes are exemplified by the Au deposition method developed in Charles Martin's laboratory $[16,17]$ for template fabrication of gold nanowires, nanotubes, and other shaped gold materials. The process involved in electroless deposition of gold can be divided in four steps:

1. "sensitization" of the membrane, during which $\mathrm{Sn}^{2+}$ ions are adsorbed by the substrate;

2. deposition of $\mathrm{Ag}$ nanoparticles by reduction of an $\mathrm{Ag}^{+}$ solution by the adsorbed $\mathrm{Sn}^{2+}$ ions;

3. galvanic displacement of the Ag particles by reduction of a $\mathrm{Au}(\mathrm{I})$ solution; and

4. catalytic reduction of more gold on the deposited $\mathrm{Au}$ nuclei, by addition of a reducing agent (formaldehyde).

A detailed description of the gold electroless deposition process may be found in the original papers [17, 34].

In contrast with electrochemical template deposition, in the electroless method the metal layer grows from the catalytic nuclei, which are located on the pore walls, toward the centre of the pores. When step 4 is stopped after a short time (e.g. 4060 min at pH 10 [23]) one can obtain hollow tubes instead of nanowires. This procedure enables the preparation of microfiltration membranes with gold pores $[35,36]$ which can be further functionalized, for instance by use of well known thiol chemistry [37], and have interesting applications as molecular sieves. A sensitive detection approach based on such modified membranes involves application of a constant potential across the membrane and measuring the drop in the trans-membrane current on the addition of the analyte. Detection limits as low as $10^{-11} \mathrm{molL}^{-1}$ have been obtained [38].

Other metals, for example $\mathrm{Cu}$ [39], $\mathrm{Pd}$ [40], and $\mathrm{Ni}-\mathrm{P}$ [41] can also be deposited in polycarbonate templates by electroless deposition. In this case the procedure must be suitable for the desired metal.

When the purpose of deposition is to obtain freestanding metallic structures it is possible to completely etch the template. Polycarbonate can be dissolved by use of organic solvents, for example $\mathrm{CH}_{2} \mathrm{Cl}_{2}-\mathrm{C}_{2} \mathrm{H}_{5} \mathrm{OH}$ mixtures [9, 42], or, as an alternative, by etching with oxygen plasma [43].

For fabrication from a metalized membrane, an easily handled electrode system, the following procedure is typical $[9,17,23,35,44-46]$.

1. Remove the outer gold layer from the smooth side of the membrane by peeling it off with adhesive tape (3 M Magic). In this way the tips of the nanowires remain exposed, under the shape of an ensemble of gold nanodiscs.

2. Attach a piece of copper adhesive tape $(5 \mathrm{~mm} \times 60 \mathrm{~mm})$ with conductive glue (Ted Pella) on a small adhesive 
non-conducting aluminium square and then attach the latter to the lower Au-coated surface of a $5 \mathrm{~mm} \times 5 \mathrm{~mm}$ piece of peeled membrane, so that only a small part is in contact with the copper tape.

3. Apply strips of non-conductive tape to the lower and upper sides of the assembly to insulate the aluminium and copper tape. This can be achieved by use of a piece of adhesive insulating tape or heat-shrinkable adhesive polymer film, for example as Topflite Monokote or similar. Note that a circular hole with an area of, typically, $0.07 \mathrm{~cm}^{2}$ is punched into the upper piece of insulator before it is placed on the assembly. The surface of the ensemble exposed to the solution defines the geometric area of the $\operatorname{NEE}\left(A_{\text {geom }}\right)$.

4. As a final step, the NEE assembly is heat-treated at $150{ }^{\circ} \mathrm{C}$ for $15 \mathrm{~min}$. This procedure produces a watertight seal between the gold nanowires and the surrounding polycarbonate.

Note that $A_{\text {geom }}$ can be changed at will [47], without affecting the signal-to-noise $(S / N)$ ratio which is typical of NEEs. Figure 4 shows a side view of an NEE ready to be used in an electrochemical experiment.

\section{Ordered arrays of nanoelectrodes by nanolithography}

Techniques such as ion beam lithography[48-50], electron beam lithography (EBL) [51], nanoimprint [52], or scanning probe lithography $[53,54]$ enable one to achieve highresolution nanostructuring, i.e. precise positioning and sizing down to a scale of a few nanometres. This spatial resolution capability has been exploited to prepare ordered arrays of nanoelectrodes [13, 48, 51]. A recent study [55] demonstrated that PC, also, can be used as a high-resolution resist for e-beam lithography. It is worth stressing that PC, in addition to its low cost, has the advantage of being suitable for easy chemical functionalization with biomolecules, by using well known functionalization procedures $[12,56]$.

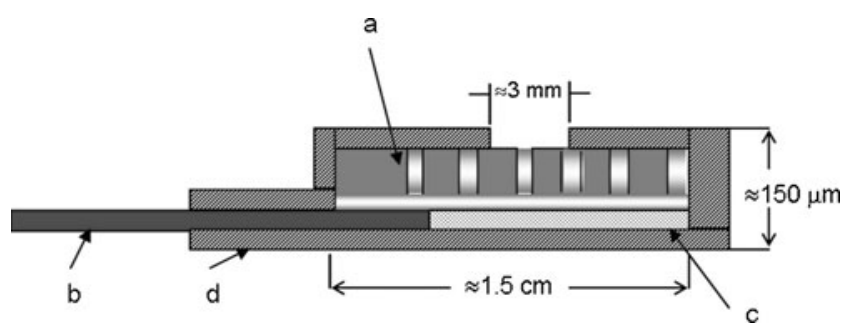

Fig. 4 Schematic representation of an NEE section, prepared by using a track-etched polycarbonate membrane as template: $(a)$ track-etched golden membrane; $(b)$ copper adhesive tape with conductive glue to connect to instrumentation; $(c)$ aluminium adhesive foil with nonconductive glue; $(d)$ insulating tape. Note: the dimensions of the pores (nanofibres) are only indicative and not to scale (reprinted, with permission, from Ref. [46])
These PC-based nanoelectrodes are fabricated by patterning arrays of holes in a thin film of $\mathrm{PC}$ spin-coated on a gold layer on $\mathrm{Si}-\mathrm{Si}_{3} \mathrm{~N}_{4}$ substrate. To improve adhesion of the $\mathrm{Au}$ film, a thin $\mathrm{Cr}$ or Ti interlayer is previously evaporated. The PC surface is exposed to the e-beam and the tracks developed (etched) in $\mathrm{KOH}$. As shown in Fig. 5, because the properties of PC enable its use as a high-resolution e-beam resist, it is possible to obtain a perfectly ordered array of nano-holes, of controlled diameter, as small as $50 \mathrm{~nm}$ [55]. These holes can be used as recessed nanoelectrodes, and by further electrochemical deposition of gold, it is possible to fill the holes partially or totally to obtain arrays of inlaid nanodisc electrodes (Fig. 6). The perfect control of the geometry of the array enables full control of diffusion in the region of the so-obtained NEA.

\section{Electrochemistry with polymer-templated electrode ensembles and arrays}

\section{Diffusion at arrays or ensembles of nanoelectrodes}

To a first approximation, an NEE or NEA can be regarded as an assembly of very small ultramicroelectrodes separated by a non-conductive substrate. An ultramicroelectrode is an electrode with at least one dimension lower than or comparable with the average thickness of the diffusion layer in a typical voltammetric experiment $(<25 \mu \mathrm{m})$. At this scale, edge effects become relevant, and diffusion from the bulk of the solution should be described by radial geometry, rather than the linear geometry typically used for larger electrodes $(>100 \mu \mathrm{m})$. Under radial diffusion conditions the voltammograms are sigmoidal in shape, and the limiting current $\left(I_{\text {lim }}\right)$, not the peak current, is the crucial condition directly related to analyte concentration.

For ultramicroelectrodes, the thickness, $\delta(t)$, of the diffusion layer around the electrode is given by Eq. (1) [57]:

$1 / \delta(t)=\left[1 /(\pi D t)^{1 / 2}\right]+1 / r$

where $D$ is the diffusion coefficient of the species, $t$ is the time of the experiment, and $r$ is the radius of the electrode.

As the electrode decreases in size, the diffusion layer thickness approaches the electrode dimensions.

The steady-state diffusion-controlled limiting current, $I$ $(t \rightarrow \infty)$, is inversely proportional to the diffusion layer thickness, in accordance with Eq. (2) [57]:

$I(t \rightarrow \infty)=n F A C^{\circ} / \delta(t \rightarrow \infty)$

where $n$ is the number of electrons exchanged, $F$ is the faraday constant, $A$ is the electrode surface area, and $C^{\circ}$ is the bulk concentration of the redox species. Dividing Eq. (2) by $A$ reveals that smaller (nano)electrodes will furnish higher 
Fig. 5 a SEM micrograph of a nanohole matrix on a PC membrane, obtained by e-beam lithography, development at $70{ }^{\circ} \mathrm{C}$ for $60 \mathrm{~s}$ and subsequent electrochemical gold deposition. b Top view of $75 \mathrm{~nm}$ radius dots in a hexagonal array on PC film; inset: higher magnification detail (reprinted, with permission, from Ref. [55])
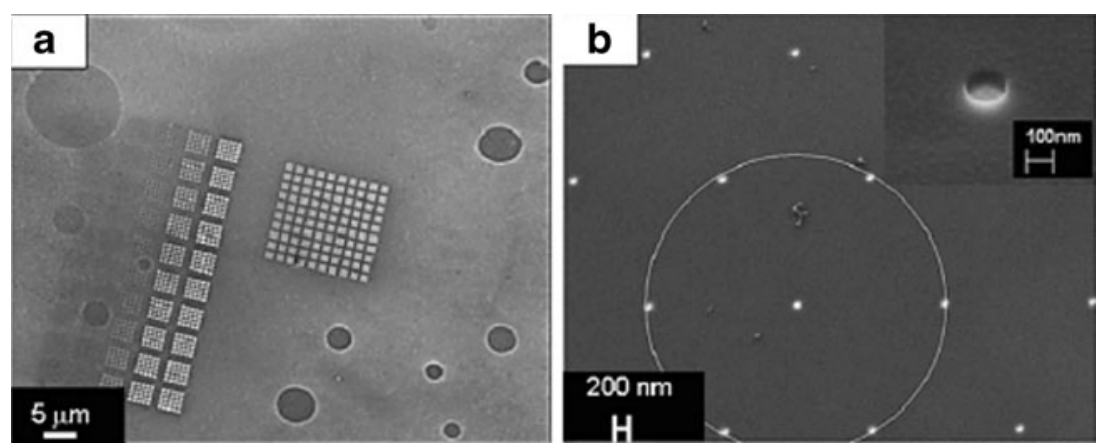

current densities as a consequence of this enhanced mass transport.

A characteristic feature of nanoelectrodes is that, when electrode dimensions are in the ten of a nanometre range, the thickness of the diffusion layer is reduced to an extent such that its dimensions is comparable with the thickness of the electrical double layer. Electrostatic forces between the ions in the double layer and the redox analyte can accelerate (retard) the flux of redox species with ionic charge opposite (equal) to the ions in the double layer, so generating the conditions for further enhancement (lowering) of mass transport to the nanoelectrode surface. Dickinson and Compton [58] recently presented a first attempt to analyse these effects, providing numerical solutions of the Poisson-Boltzman equation, calculated for hemispherical nanoelectrodes of vanishing size. Their study revealed significant effects of curvature on the diffuse double-layer profiles, which become relevant for electrodes with radii less than $50 \mathrm{~nm}$, even in the presence of supporting electrolyte. An enhanced driving force is therefore expected for nanoelectrodes as compared with electrodes larger than 50-100 nm [58]. Further studies, expected to go deeper into theoretical modelling of electrochemical processes at nanoelectrodes are, therefore, urgently required [59].
From perspective of diffusion, the voltammetric responses of NEEs/NEAs can vary, depending on the scan rate or the reciprocal distance among the nanoelectrodes [60-62]. The different limit situations are summarized in Fig. 7. When radial diffusion boundary layers totally overlap, i.e. when the diffusion hemisphere is larger than the mean hemidistance among the nanoelectrodes, NEEs behave as macroelectrodes with regard to the Faradic current (total overlap, peak shape voltammograms, case V). When the diffusion hemisphere becomes shorter (higher scan rates) or the hemidistance among nanodiscs is larger, the voltammetric response is dominated by radial diffusion conditions at each element (pure radial conditions, sigmoidally shaped voltammograms, case III). At very high scan rates, the linear active state is reached (case I) in which linear diffusion predominates at each nanodisc (peak-shaped voltammograms, but with peak currents much smaller than case V). Obviously, intermediate situations can be observed (cases IV and II).

Recent theoretical studies [60, 63-66], examined in detail the effect of the different diffusion conditions on the voltammetric responses recorded at arrays of ultramicro and nanoelectrodes. In particular, Guo and Lindner [63] introduced a very useful zone diagram in which the combination of suitable
Fig. 6 SEM images of NEAs with holes $500 \mathrm{~nm}$ in diameter with gold electrochemically deposited inside for $0 \mathrm{~s}(\mathbf{a}), 10 \mathrm{~s}$ (b), $20 \mathrm{~s} \mathrm{(c),} \mathrm{and} 30 \mathrm{~s} \mathrm{(d).}$

Estimated recession depths: (a) $450 \mathrm{~nm}$; (b) $300 \mathrm{~nm}$; (c) $150 \mathrm{~nm}$; (d) $0 \mathrm{~nm}$ (reprinted, with permission, from Ref. [55])
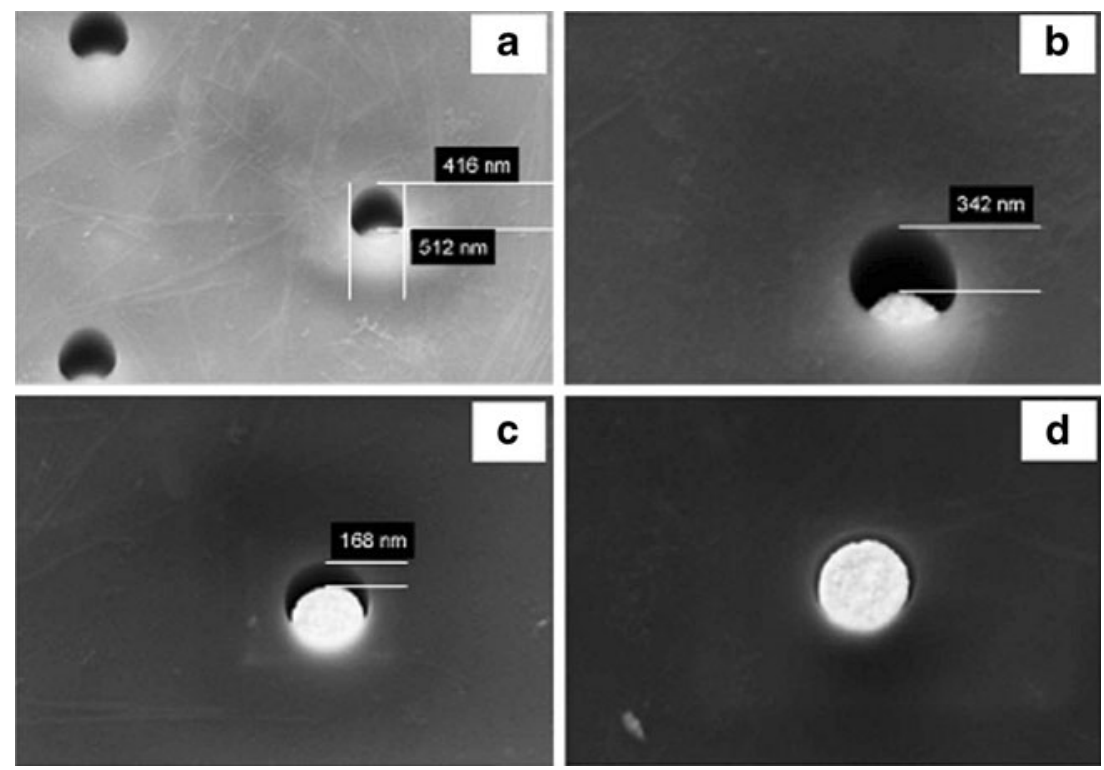
Fig. 7 Simulated concentration profiles, with isoconcentration contour lines, over a microelectrode array representing the five main categories of diffusion modes (forms I to V). In the scale bar next to the figure, the red colour represents the bulk concentration and the blue colour represents zero concentration. The second scale bar represents a relative concentration scale for the contour lines. Typical CVs of the each category are shown at the right (reprinted, with permission, from Ref. [63])
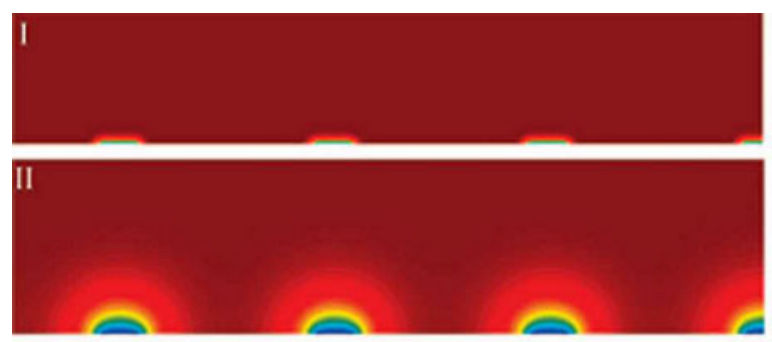

III

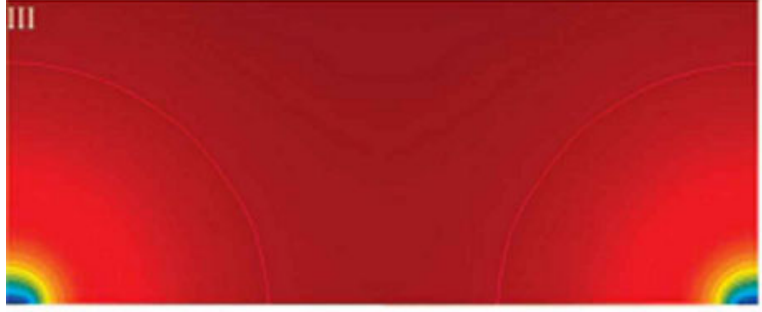

IV
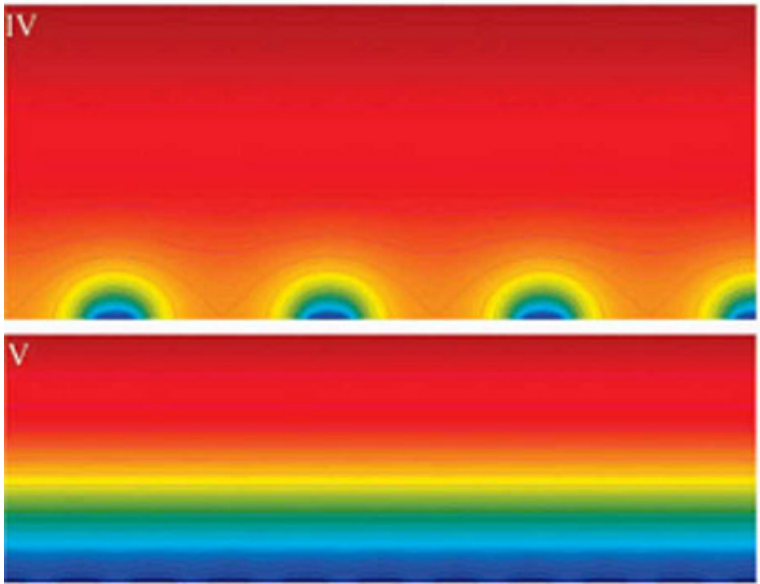

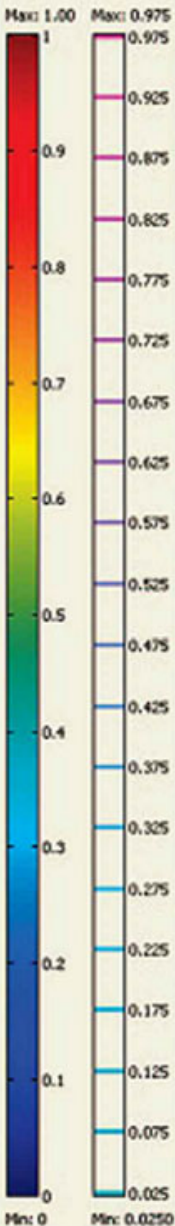

dimensionless variables enables one to determine the diffusion conditions (and the kind of voltammetric response) in operation for a specific type of array, at a specific voltammetric scan rate (Fig. 8). The study focused on arrays of microelectrodes but can be extended to arrays of nanoelectrodes.

Note also that this simulation was developed for arrays in which the effects at the border of the array are negligible, i.e. for arrays including a very large number of electrodes [47, $55,60]$. This condition can be achieved for arrays of small size only if electrode size is very small, i.e. at the nanoscale level. The following example can aid understanding of this concept. If one builds an array of 100 electrodes ordered according to a square geometry, 36 of the electrodes will be on the periphery. This means border effects will be relevant for at least $36 \%$ of the electrodes. If one wishes to make such border effects negligible, it is necessary to increase the overall number of electrodes in the array by two orders of magnitude; e.g., in a $10^{4}$-electrode (100 squared) array only $3.96 \%$ of the electrodes will be on the perimeter.

A distance between electrodes of $10 \times r$, where $r$ is the radius of the individual electrodes, is sufficient to prevent cross-talk between the electrodes [60]. This means that if $r=$ $10 \mu \mathrm{m}$, the side of a $10^{4}$-electrodes array will be as large as
$1 \mathrm{~cm}$. If, however, $r=0.1 \mu \mathrm{m}$, the side of the array (with the same number of electrodes) will be reduced to $1 \mathrm{~mm}$. This is particularly important for electrochemical biosensors, for

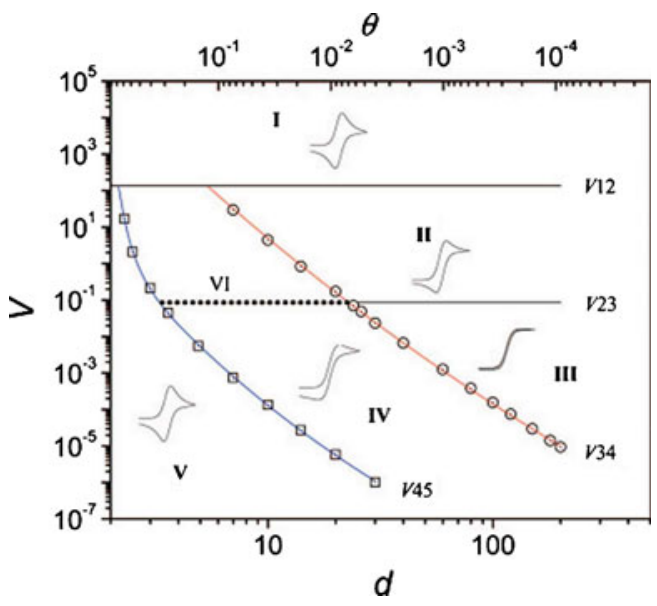

Fig. 8 Zone diagram of cyclic voltammetric behaviour at microelectrode arrays. $d$ is the centre-to-centre distance of individual electrodes in the array (measured in units of a), $V$ is the dimensionless scan rate, and $\theta$ is the fraction of electrochemically active area in the array (reprinted, with permission, from Ref. [63]) 
which immobilization of expensive biomolecules on the surface of the electrode is needed and miniaturization is essential.

For arrays composed of a small number of nanoelectrodes, however, border effects are relevant [13]. Under these conditions, when the overall size of the array is in the micrometre range, even for arrays operating under total overlap conditions sigmoidally shaped voltammograms are observed [13].

\section{Current signals at NEEs}

Total overlap diffusion is usually observed for NEEs fabricated from commercially available track-etched membranes [17]. Transition from these conditions as a function of nanoelement distance has, nevertheless, been demonstrated experimentally by use of specially-made membranes [61]. It has recently been shown that, for NEE, transition from the total overlap to the pure radial diffusion can be observed on increasing electrolyte viscosity [67]. The voltammetric patterns recorded at NEEs in high-viscosity ionic liquids are, indeed, peak-shaped $\mathrm{CV}$ at low scan rates but become sigmoidally shaped at high scan rate (Fig. 9).

Note that the diffusion coefficient, $D$, decreases with increasing viscosity, so that diffusion hemispheres around each nanoelectrode are smaller in high-viscosity medium.

Returning to the more common situation of the voltammetric use of NEEs in aqueous media, it is worth stressing that, for electroanalytical purposes, the main advantage of total overlap diffusion is the improved detection limit compared with conventional electrodes with the same surface area. This is because, for NEEs, operating under total overlap diffusion conditions, the Faradaic current $\left(I_{\mathrm{F}}\right)$ is proportional to the total geometric area of the ensemble exposed to the sample solution ( $A_{\text {geom }}$, area of the nanodiscs plus insulator area) whereas the double layer capacitive current $\left(I_{\mathrm{C}}\right)$, which is the main

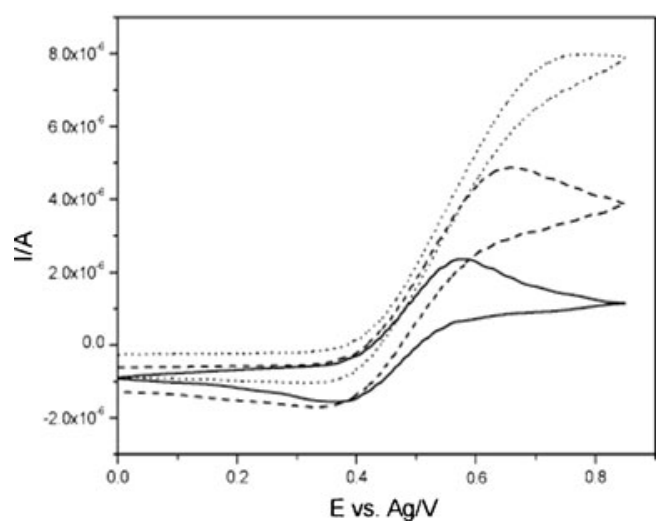

Fig. 9 Cyclic voltammograms, recorded at different scan rates, at an NEE (geometric area $0.07 \mathrm{~cm}^{2}$; active area $0.004 \mathrm{~cm}^{2}$ ), $50 \mathrm{mmolL}^{-1}$ ferrocene in [tris $(n$-hexyl)tetradecylphosphonium][bis(trifluoromethylsulfonyl)amide]. Scan rates: full line $5 \mathrm{mV} \mathrm{s}^{-1}$; dashed line $50 \mathrm{mV} \mathrm{s}^{-1}$; dotted line $500 \mathrm{mV} \mathrm{s}^{-1}$ (reprinted, with permission, from Ref. [67]) component of the noise in electroanalytical chemistry, is proportional to the nanodisc area only (active area, $A_{\text {act }}$ ) [17].

Typical values of the geometric area range from 0.008 to $0.580 \mathrm{~cm}^{2}$ [47]; this property is defined at the moment of fabrication of the NEE from the dimension of the hole punched into the insulator. The active area can be easily calculated from membrane characteristics such as pore density $(q)$ and mean pore radius $(r)$, by use of the equation:

$A_{\text {act }}=\pi r^{2} q A_{\text {geom }}$

The ratio of the active area to the geometric area defines a key property named the fractional electrode area $(f)$ :

$f=A_{\text {act }} / A_{\text {geom }}$

Faradaic-to-capacitive current ratios at NEEs and conventional electrodes with the same geometric area are related by Eq. (5) [68]:

$\left(I_{F} / I_{C}\right)_{N E E}=\left(I_{F} / I_{C}\right)_{c o n v} f$

Because typical $f$ values for NEEs are between $10^{-3}$ and $10^{-2}, I_{\mathrm{F}} / I_{\mathrm{C}}$ ratios at NEEs can be $2-3$ orders of magnitude higher than at conventional electrodes with the same geometric area. This improvement of the Faradaic to capacitive currents ratio explains why detection limits (DLs) at NEEs can be 2-3 orders of magnitude lower than for conventional electrodes [17, 68-70]. Because improvement of $S / N$ ratios are strictly related to fractional area, the electroanalytical performance of NEEs is not affected by any variation in the geometric area as long as the active area changes accordingly, i.e. $f$ is kept constant [47]. Because the main advantage of NEEs over conventional macro (mm-sized) or even ultramicro ( $\mu \mathrm{m}$-sized) electrodes is a dramatic lowering of double-layer capacitive currents [17, 69], if it is not possible to directly characterize the morphology of the electrodes, the lack of this characteristic should be taken into account to discriminate well-prepared from defective NEEs.

For example, voltammograms affected by a large capacitive current, are characteristic of poor sealing between the nanowires and the surrounding PC insulator and/or heavy scratching of the PC membrane caused by improper handling of the NEE. On the other hand, a radial diffusive contribution to the overall signal suggests a larger distance between the nanoelectrodes, possibly because of only partial filling of the pores with gold [46].

\section{Electron-transfer kinetics}

An important feature characterizing NEEs and NEAs is that their responses are very sensitive to electron-transfer kinetics [17]. According to model proposed by Amatore et al. [71], and to more recent theoretical models [63-65], an NEE behaves as a partially blocked electrode (PBE) whose 
current response is identical with that of a naked electrode of the same overall geometric area, but with a smaller apparent rate constant $\left(k_{\text {app }}^{\circ}\right)$ for the electron transfer which decreases as the coverage of the surface increases. According to this model, the nanodisc electrodes are the unblocked surface and the template membrane is the blocking material.

The apparent rate constant $\left(k_{\text {app }}^{\circ}\right)$ is related to the true standard rate constant by the equation:

$k_{\text {app }}^{\circ}=k^{\circ}(1-\vartheta)=k^{\circ} f$

where $\vartheta=\left(A_{\text {geom }}-A_{\text {act }}\right) / A_{\text {geom }}$ and $f$ is the fractional electrode area (Eq. 4).

From an analytical perspective, Eq. (6) means that high Faradaic peak currents are observed at NEEs only for redox couples with "very reversible" behaviour. In cyclic voltammetry $(\mathrm{CV})$, in fact, the reversibility of a redox system depends on the $k^{\circ}$ value and on the scan rate $(v)$. For conventional electrodes, reversible patterns are obtained when:

$v^{1 / 2} \leq\left(k^{\circ} / 0.3\right)$

but if NEEs are used, $k^{\circ}$ is substituted by $k_{\text {app }}^{\circ}$, and Eq. (7) becomes:

$v^{1 / 2} \leq\left[\left(k^{\circ} f\right) / 0.3\right]$

Considering that mean $f$ values range from $10^{-2}$ to $10^{-3}$, from Eq. (8) we can conclude that, for a specific redox couple, the scan rate that defines the transition between reversible and quasi-reversible behaviour will be $2-3$ orders of magnitude lower than those for conventional electrodes. Note that such a boundary scan rate will decrease with decreasing $f$. This limitation must be seriously taken into account when trying to optimize NEEs for bioanalytical application, because it is important to consider the contrasting effect both of the increased $I_{\mathrm{F}} / I_{\mathrm{C}}$ value and the apparent slowing down of the electron-transfer kinetics. Mechanistically, however, this is an advantage, because it means that with NEEs it is easier to measure very large $k^{\circ}$ values experimentally [57]. By analysis of the dependence of $\Delta E_{\mathrm{p}}$ on scan rate [72], and use of suitable working curves [73], smaller $k_{\text {app }}^{\circ}$ values are obtained and converted to larger $k^{\circ}$ by use of Eq. (6) [71].

\section{Current signals at nanolithographed NEAs}

As already explained, use of advanced nanolithographic methods enables the preparation of ordered arrays of nanoelectrodes with controlled geometry. The effect of the distance between and radius of the nanoelectrodes, and of their number (with regard to negligible border effects) has been explained above. For NEA, the ability to control the geometry of the electrodes in the array enables one to obtain electrode arrays which operate under pure radial control rather than under total overlap conditions (Fig. 10).

However, it is worth stressing that, because of the nanolithographic process itself, quite often the nanoelectrodes obtained are slightly recessed, so that theoretical model for such geometry must be taken into account $[50,55]$.

\section{Bioelectroanalysis with arrays and ensembles of nanoelectrodes}

NEEs and NEAs can both be used for interesting bioanalytical applications. However, it should be noted that NEAs with reliable electroanalytical characteristics have been described only very recently, and, consequently, few examples of practical applications have yet been described. For instance, only very recently Triroj et al. [74] described a microfluidic chip which uses NEAs as for miniaturized detection of prostatespecific antigen. Arumugam et al. [15] described a robust and scalable wafer-scale method for fabrication of multiplexed biosensors. Each sensor chip consists of nine individually addressable arrays that use electron beam-patterned vertically aligned carbon fibres as the sensing element.

The "electroanalytical story" of NEEs is longer (starting from 1995 [17]) and, therefore, richer with examples of bioanalytical applications.

The improved $S / N$ ratio typical of NEEs makes them particularly suitable for direct determination of electroactive species at low concentrations. Besides application to tracing reversible redox mediators used in biosensors, for example ruthenium complexes or ferrocene derivatives [17, 69], phenothiazines, methylviologen, and others [70], NEEs have proved

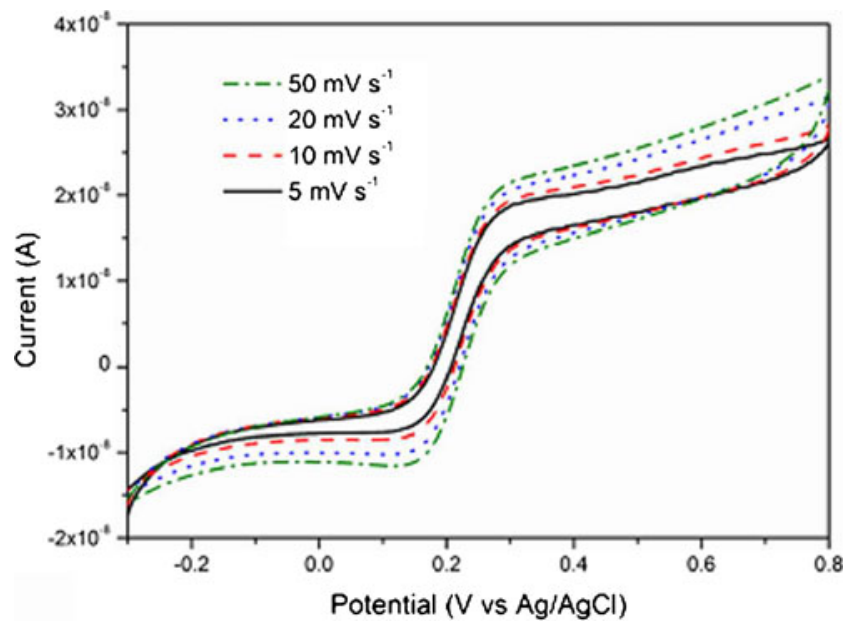

Fig. $10 \mathrm{CVs}$ recorded in $10^{-4} \mathrm{molL}^{-1}$ ferrocene methanol and $0.5 \mathrm{~mol}$ $\mathrm{L}^{-1} \mathrm{NaNO}_{3}$. Scan rates: 5 (full line), 10 (dashed line), 20 (dotted line), and $50 \mathrm{mV} \mathrm{s}^{-1}$ (dash-dot line). Geometrical characteristics: nanodisc radius $=75 \mathrm{~nm}$, distance centre to centre $=3 \mu \mathrm{m}$, estimated number of nanoelectrodes in the array $=1.1 \times 10^{-4}$ (reprinted, with permission, from Ref. [55]) 
useful for voltammetry of more electrochemically complex systems, for example the heme-protein cytochrome $c$ [75]. In this instance well resolved voltammograms were obtained for submicromolar concentrations of the protein $[47,75]$ both with and without promoters, for example 4,4'-bipiridyl typically used to promote cytochrome $c$ electrochemistry [76-79]. These promoters are generally required to avoid adsorption and/or denaturation $[80,81]$ of cytochrome $c$ on the Au surface. However, such adsorption is concentration-dependent so reducing the cytochrome $c$ solution concentration below the adsorption limit (possible with NEEs because of their lower detection limit) can overcome adsorption-related problems.

\section{NEE and NEA-based biosensors}

Direct detection strategies are not always feasible, especially for more complex or non-electrochemically active biomolecules. For this reason, in typical schemes used for electrochemical biosensors, a biorecognition layer is immobilized directly on the electrode surface and the signal is produced by exchange of electrons with the underlying electrode. This method has also been applied to arrays of nanoelectrodes in which the nanodiscs are used both for transduction of the signal and adsorption of the active biomolecules [82].

Many of the advantages of use of NEEs and NEAs for biodetection come from the enhanced mass fluxes which characterize these electrode system. As a consequence, such improvements are expected to be negated for systems in which the redox species are adsorbed on the surface of the nanoelectrodes, because, for these, the electrochemical signal does not depend on a diffusion-controlled process. For this reason, the best architecture for NEEs and NEAs is that in which the biorecognition element is adsorbed on the nanoelectrode or on the insulating polymer close to the nanoelectrode, and electroactive species, for example the substrate and/or the redox mediator, diffuse in the solution so that a diffusional pathway controls the electron-transfer process. This situation is achieved when:

1. electron transfer occurs between an enzyme immobilized on the surface of the nanoelectrodes and a diffusing substrate; and

2. the biorecognition layer is immobilized in the vicinity of the nanoelectrodes (e.g. on the PC of the NEE [12]) and a diffusing redox mediator shuttles electrons between this layer and the neighbouring nanoelectrodes.

In such arrangements, the enhanced mass transport typical of nanoelectrodes is fully exploited. In the following text we discuss some examples of these approaches.

Functionalization of the nanoelectrodes: from 2D to 3D NEEs

For highly miniaturized electrodes, for example NEEs, the number of biomolecules immobilized on the nanoelectrodes is often very small, resulting in poor current signals. A possible means of controlled increase of the active area of NEEs is partial etching of the polycarbonate template membrane. This procedure causes the structure of the final ensemble to change from a flat 2D surface made of metal nanodiscs embedded in a non-conductive substrate to a $3 \mathrm{D}$ structure made of an ensemble of nanowires partially protruding from the insulating layer. Three-dimensional NEEs have been obtained from 2D NEEs by two different methods. The first, proposed by Martin et al. [43], exploits a $\mathrm{O}_{2}-\mathrm{Ar}$ plasma for controlled etching of the templating polymer, exposing approximately $200 \mathrm{~nm}$ of gold nanowires. A simpler method, proposed by Zoski [42], is based on substituting the plasma-etching with chemical etching, by using suitable solvents to partially dissolve the polycarbonate. The best mixture for a controlled rate of etching was found to be $50: 50 \mathrm{CH}_{2} \mathrm{Cl}_{2}-$ $\mathrm{C}_{2} \mathrm{H}_{5} \mathrm{OH}$. This method was used by De Leo et al. [9] to develop a glucose sensor based on use of a nitrofluorenone mediator (bound on the gold nanowires of a 3D NEE) which exchanges electrons with NADH-dependent glucose dehydrogenase.

Cao et al. [83, 84] used the enhanced active surface area of 3D NEEs for detection of the chemotherapeutic agent Daunorubicin. In this approach the analyte is adsorbed on the surface of the gold nanowires and analysed directly by SWV, resulting in an LOD as low as $8.9 \times 10^{-8} \mathrm{molL}^{-1}$ $(S / N=3)$ [84]. Functionalization of the nanowires with Lcysteine increased the amount of adsorbed analyte, reducing the detection limits to $1.0 \times 10^{-8} \mathrm{molL}^{-1}(S / N=3)$ [83].

Three-dimensional NEEs have also been used as sensitive biosensors for detection of DNA hybridization $[85,86]$. Single-stranded DNA can be immobilized both on the gold nanorods surface of the 3D NEEs $[85,86]$ or on the polymer membrane surface [87]. In the former, the detection mechanism exploits an electrocatalytic reaction between a primary acceptor, namely $\mathrm{Ru}\left(\mathrm{NH}_{3}\right)_{6}{ }^{3+}$, and a secondary acceptor, namely $\mathrm{Fe}(\mathrm{CN})_{6}{ }^{3-}$. The first ion is reduced at the electrode surface and then reoxidized by excess of the anion, resulting in a catalytic electrochemical process. By increasing the concentration of negatively charged phosphate groups at the Au surface of NEEs, by hybridization with complementary sequences, the local concentration of $\mathrm{Ru}\left(\mathrm{NH}_{3}\right)_{6}{ }^{3+}$ also is increased (Fig 11).

Functionalization of the gold surface on a 3D NEE has also been applied to determination of the ovarian cancer marker mucin-16 (MUC-16) [1]. Viswanathan et al. developed an electrochemical immunosensor using ferrocene carboxylic acid-encapsulated liposomes bonded with monoclonal antimucin-16 antibodies ( $\alpha$ MUC16). $\alpha$ MUC16 were also immobilized on a self-assembled monolayer of cysteamine on the 3D NEE obtained via cross-linking with carbodiimide (EDC) and $N$-hydroxysulfosuccinimide (Sulfo-NHS). A sandwich immunoassay was performed on $\alpha$ MUC16-functionalized 3D NEE with MUC16 and immunoliposomes. Differential 


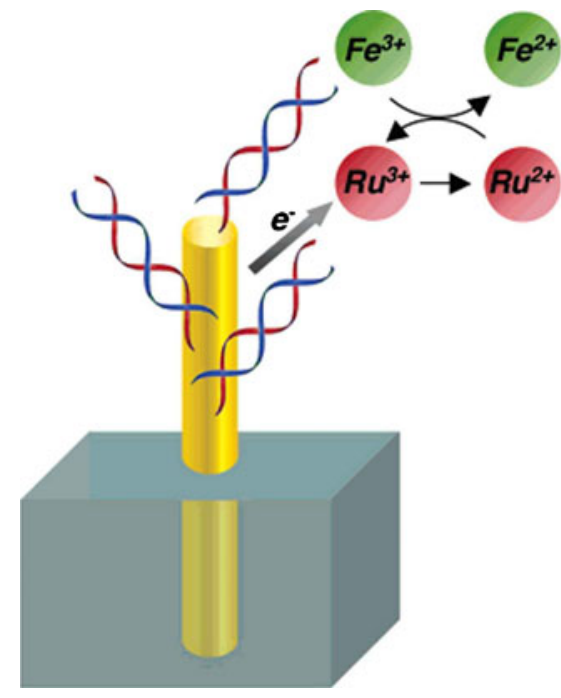

Fig. 11 Schematic illustration of $\mathrm{Ru}(\mathrm{III}) / \mathrm{Fe}(\mathrm{III})$ electrocatalysis at a DNA-modified Au NEE (reprinted, with permission, from Ref. [85])

pulse voltammetry was used to quantify the faradic redox response of ferrocene carboxylic acid released from immunoliposomes and to quantify the MUC-16 concentration (Fig. 12). The detection limit was $5 \times 10^{-4} \mathrm{U} \mathrm{mL}^{-1}(S / N=3)$.
In some cases, immobilization of biomolecules directly on the surface of the nanoelectrodes could hinder the electron transfer and reduce sensitivity; however Viswanathan et al. [1] demonstrated that for detection of the ovarian cancer marker MUC16 , an optimum amount of immobilized bioreceptor molecule can be found to make this negative effect negligible. The results obtained with this immunosensor were in good correlation with a commercial ELISA test performed on the same samples, proving functionalized 3D NEEs were a viable alternative, especially for the development of home testing kits.

Alternative designs: gold nanoparticles on NEEs

Use of etched 3D NEEs to increase the amounts of biomolecules adsorbed on gold nanowire surfaces proved to be a viable process, although with the drawback of an increase of the capacitive current and, consequently, an increase of the $S / N$ ratio [9].

One way of reducing this drawback has recently been proposed [88] — increasing the nanoelectrode area not by etching the templating polymer but depositing gold nanoparticles on the gold nanodisc electrodes. The gold nanoparticles (AuNPs) are immobilized on the surface of NEEs

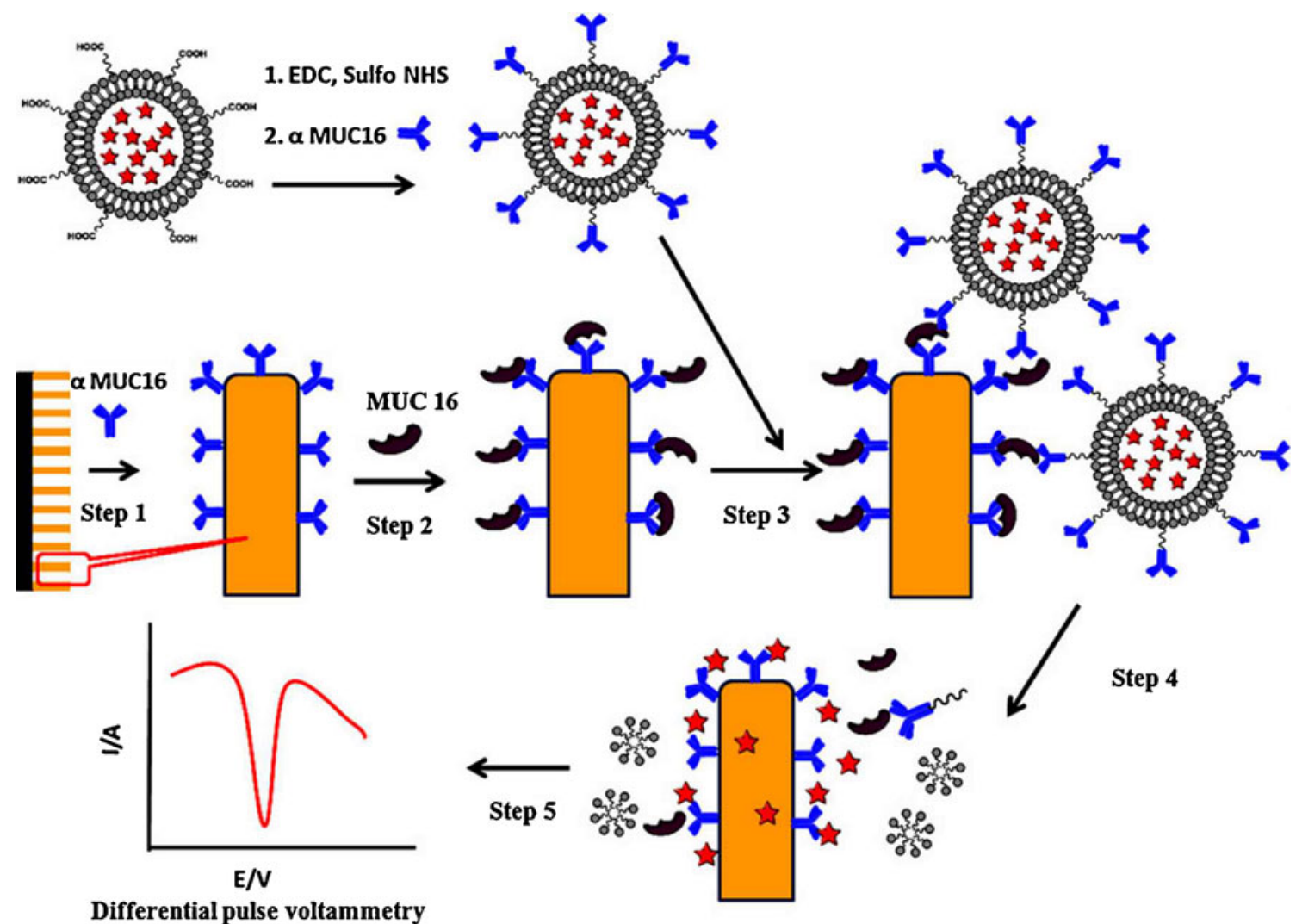

Fig. 12 Sketch of the electrochemical MUC-16 detection method. Step 1: $\alpha$ MUC16 immobilized on the 3D NEE exposed wires. Step 2: MUC-16 immunoconjugated with the antibody on the surface of the nanowires. Step 3: sandwich immunocomplex with immunoliposomes.

Step 4: disruption of immunoliposomes and release of the redox species, whose concentration is determined by SWv (Step 5) (reprinted, with permission, from Ref. [1]) 


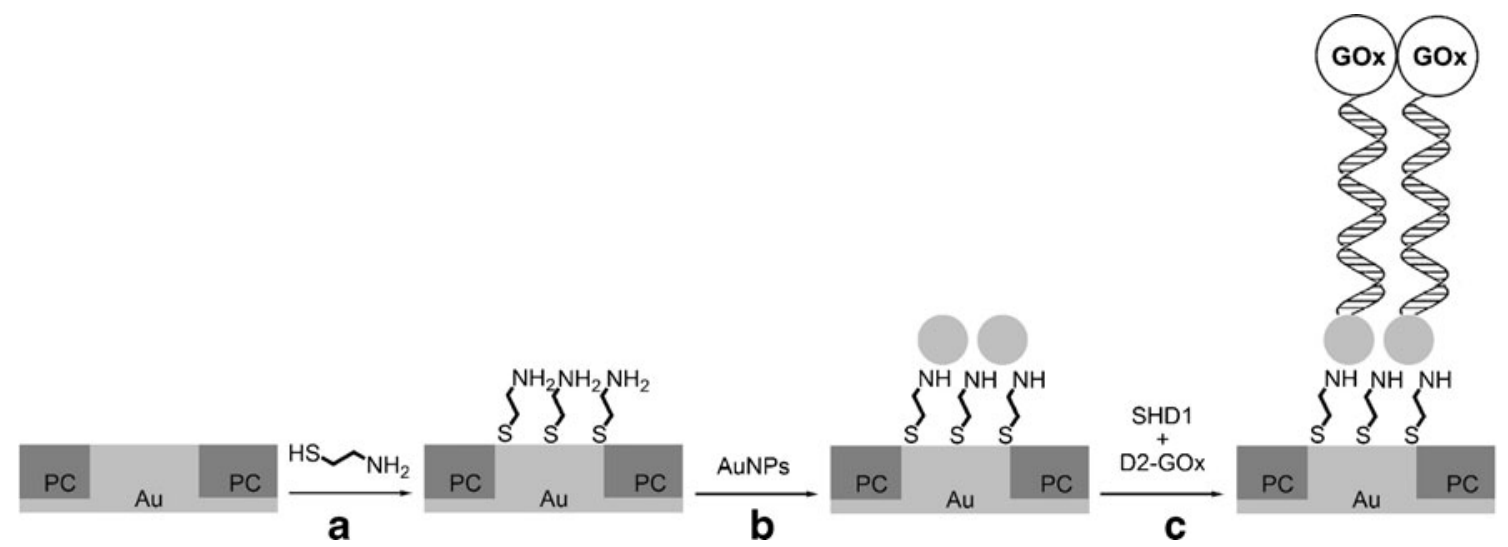

Fig. 13 Schematic illustration of the target ssDNA detection mechanism by a so called AuNPs-NEE: $(a)$ modification of NEE with cysteamine; $(b)$ immobilization of gold nanoparticles; $(c)$ functionalization with probe sequences (SHD1) and subsequent hybridization

by using cysteamine as a cross-linker able to bind the AuNPs to the heads of the nanoelectrodes, to obtain a socalled AuNPs-NEE. Analysis of cyclic voltammograms recorded in pure supporting electrolyte showed that the presence of the nanoparticles resulted in an approximately tenfold increase in the electrochemically active area of the ensemble. Measurement of the amount of electroactive polyoxometalates which can be adsorbed on the gold surface of NEEs vs. AuNPs-NEEs confirmed a significant increase of active area for the latter. This evidence indicates there is a good electronic connection between the AuNPs and the underlying nanoelectrodes. The possibility of exploiting AuNPs-NEEs for biosensing applications was tested for DNA-hybridization detection. After immobilization on the gold surface of AuNPs-NEEs of a thiolated single-stranded DNA, hybridization with complementary sequences labelled with glucose oxidase (GOx) was performed (Fig. 13). Detection of the hybridization was achieved by adding the GOx substrate (i.e. glucose) and a suitable redox mediator (i.e. the (ferrocenylmethyl)trimethylammonium $\left(\mathrm{FA}^{+}\right)$cation) to the electrolyte solution; when hybridization occurs, an electrocatalytic increase of the oxidation current of $\mathrm{FA}^{+}$is recorded. Comparison of the electrocatalytic current recorded at DNA-modified NEEs and AuNPs-NEEs indicates, for the latter, a fourfold increase in sensitivity in the detection of DNA-hybridization.

Exploiting the templating membrane for functionalization purposes

To keep the $S / N$ ratio as high as possible by keeping the active area as low as possible, a different approach has recently been proposed in which the biorecognition element is immobilized on the polymeric matrix of a 2D ensemble of nanodiscs [12], and not on the metal surface of the nanoelectrodes. In such a design, transducer and biorecognition elements do not overlap with complementary target conjugated with GOx (D2-GOx). Note: the dimensions are not to scale (reprinted, with permission, from Ref. [88])

but are integrated in strict proximity, at the nanoscale level. This approach, besides maintaining excellent detection limits of 2D NEEs, should greatly increase the amount of immobilized biomolecules, without requiring etching of the template or deposition of AuNPs. The polymer surface of the templated NEE is, indeed, 2-3 orders of magnitude larger than the gold surface of the NEE. Exploiting this idea, Pozzi Mucelli et al. [12] proposed an immunosensor for determination of the human epidermal growth factor receptor HER2 in which a specific capture agent is bound to the templating PC of a NEE. In some cancers, notably some breast cancers, HER2 is overexpressed, and causes cancer cells to reproduce uncontrollably. The mechanism for detection of this protein is as follows. First, the monoclonal anti-HER2 antibody trastuzumab (commercial name Herceptin) is immobilized on the polycarbonate of a NEE. The functionalized NEE is then incubated with the sample to capture the target protein HER2. Finally, the captured protein is reacted with a primary antibody (monoclonal CB-11) and a secondary antibody, labelled with horseradish

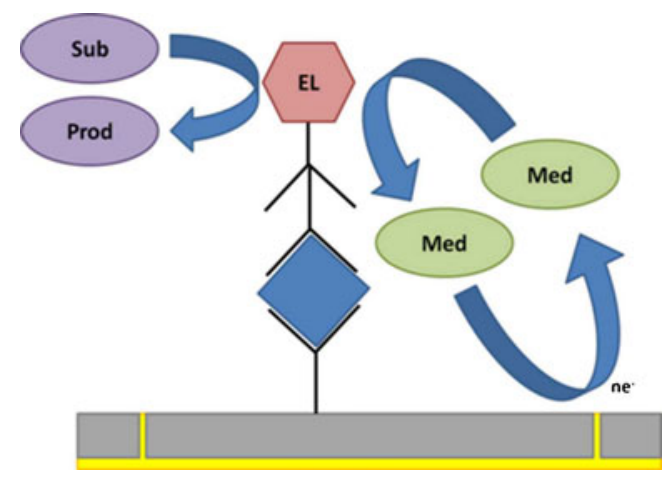

Fig. 14 Schematic illustration of the HER2 detection mechanism. A specific antibody is first attached to the polycarbonate to capture the target protein (blue square). A primary antibody binds to the protein and, subsequently, a secondary antibody tethered with the enzyme label (EL) 


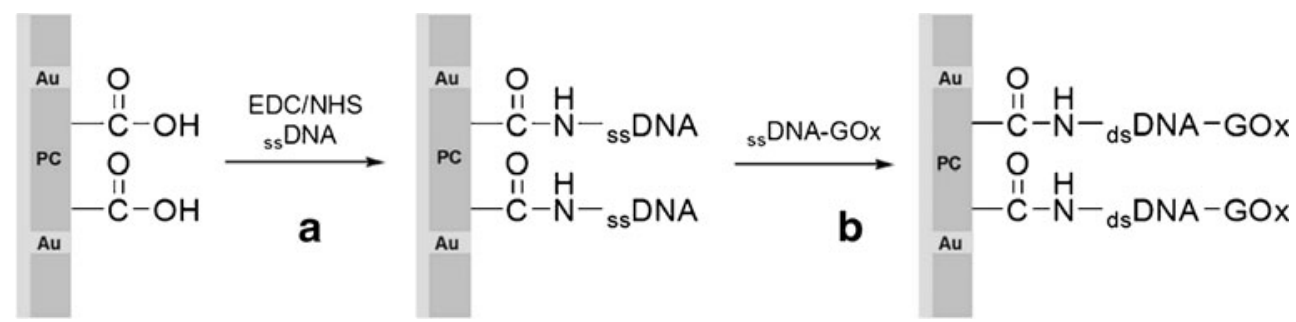

Fig. 15 Design of DNA hybridization sensor based on NEE assembly: (a) activation of $-\mathrm{COOH}$ groups of the $\mathrm{PC}$ surface and immobilization of the capture amino-end DNA probe on to the activated carboxylic

peroxidase (HRP). The biosensor is then dipped into phosphate buffer electrolyte containing the HRP substrate (i.e. $\mathrm{H}_{2} \mathrm{O}_{2}$ ) and the redox mediator methylene blue, which shuttles electrons from the nanoelectrodes to the active site of HRP (Fig. 14). A similar approach has also been applied to the electrochemical detection of fragmented antibodies, for example single chain fragment variable proteins [12].

In some cases non-specific adsorption of the proteins on the gold nanodiscs surface has been observed; this limits the electrochemical signal and, consequently, detection efficiency. To overcome this problem it is possible to protect the nanoelectrodes with a self-assembled monolayer (SAM) of short-chains thiols. This functionalization prevents protein fouling of the NEEs, enabling detection of well resolved voltammograms of the probe molecule [11]. To characterize the structures obtained, careful atomic force microscopy (AFM) characterization of the NEEs was performed.

Recently, biorecognition probes were immobilized on to the PC of NEEs for detection of DNA hybridization [87]. Single-stranded amino-terminated DNA probes (ssDNA) were bound to the $\mathrm{PC}$ by exploiting the reactivity of the carboxyl groups present on the polycarbonate surface. Titrations with thionin acetate revealed that a surface concentration of $-\mathrm{COOH}$ of the order of $9.7 \times 10^{-10} \mathrm{molcm}^{-2}$ is present on row PC; the surface concentration of carboxyl groups can be increased to $3.4 \times 10^{-9} \mathrm{molcm}^{-2}$ by controlled oxidation with $\mathrm{KMnO}_{4}$. The reactions used for the immobilization [87] are summarized in Fig. 15.

NEEs functionalized with the DNA probe are then hybridized with the target ssDNA labelled with glucose oxidase (GOx) [89]. The occurrence of the hybridization event is detected by adding, to the supporting electrolyte, excess glucose as the substrate and the ferrocenyltrimethylammonium cation as suitable redox mediator. In the event of positive hybridization, an electrocatalytic current is detected. In the proposed sensor, biorecognition and signal transduction occur in different but neighbouring sites, i.e. the PC surface and the nanoelectrodes, respectively; these sites are separated, albeit in close proximity on a nanometre scale (Fig. 16). The proposed biosensor has high selectivity and sensitivity, with the capability of detecting a few picomoles of target DNA. functionalities; (b) hybridization of DNA-GOx conjugate on to modified PC surface (reprinted, with permission, from Ref. [88])

\section{Conclusion}

Nanoelectrode ensembles and arrays can be obtained both by "bottom-up" and "top-down" nanotechnology. A typical "bottom-up" method is membrane templated deposition of ensembles of nanoelectrodes in self-standing track-etched polymer membranes. A typical "top-down" procedure, on the other hand, is fabrication of arrays of both recessed or inlaid nanoelectrodes by using advanced nanolithographic techniques, for example ion-beam or electron-beam lithography. In both cases, the final result is a composite material in which nanowires or nanodiscs of a metal conductor are embedded in a polymer matrix. Control of the geometry of the composite enables one to obtain functional materials with unique electroanalytical characteristics.

NEEs and NEAs are characterized by enhanced mass fluxes and dramatic enhancement of the signal-tobackground current ratio compared with other electrode systems. A disadvantage is their extreme sensitivity to electron-

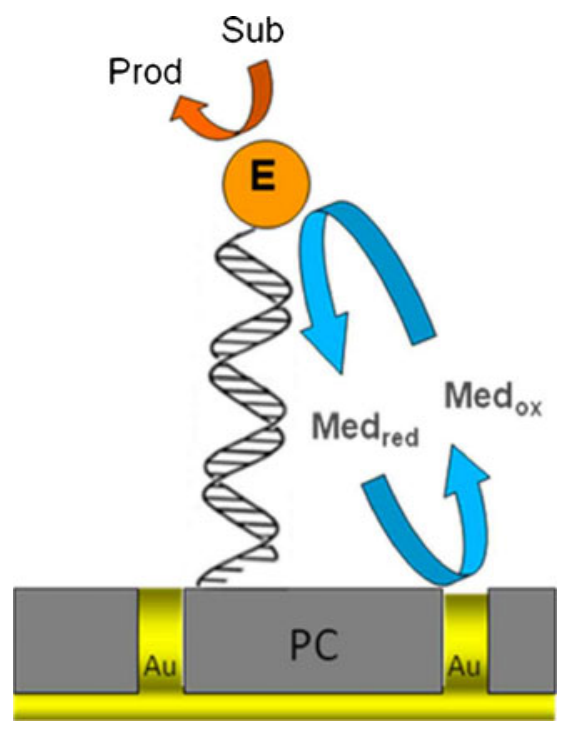

Fig. 16 Schematic illustration of two DNA biorecognition systems The probe DNA strand is first attached to the polymer membrane; the target GOx-conjugated strand is then hybridized. The mediator reacts with the reduced enzyme and gives an electrochemical signal at the nanoelectrodes 
transfer kinetics. Moreover, the small active area can limit the amount of biorecognition molecules which can be immobilized with the purpose of obtaining suitable biosensors. The first limit can be overcome by using NEEs and NEAs in electrochemical biosensors in which electron-transfer processes are determined by the electrochemistry of suitably reversible mediators. For the second problem, the active area can be suitably increased by controlled etching of the polymeric membrane or by immobilization on the nanoelectrodes of metal nanoparticles. An alternative approach is the possibility of immobilizing the biorecognition layer on the insulating polymer which surrounds the nanoelectrodes, rather than on the nanoelectrodes themselves.

Future research effort should be devoted to the development of singly addressable electrodes or of groups of nanoelectrodes. The possibility of moving from current NEEs/ NEAs (in which all nanoelectrodes are interconnected) to more sophisticated nanoelectrode systems, in which multiple analyte determination is achieved, and the extreme miniaturization of such devices, would be particularly suitable for sensors to be used in bioanalysis, both for "in vitro" and "in vivo" analysis. The advantages of multianalyte sensors are obvious for related fields, for example environmental or food analysis.

Acknowledgments The authors would like to thank MIUR (Rome) (project: PRIN 2008MWHCP2) for partial financial support.

\section{References}

1. Viswanathan S, Rani C, Delerue-Matos C (2012) Ultrasensitive detection of ovarian cancer marker using immunoliposomes and gold nanoelectrodes. Anal Chim Acta 726:79-84

2. Shi HB, Yeh JI (2007) Part I: Recent developments in nanoelectrodes for biological measurements. Nanomedicine-UK 2:587-598

3. Arredondo M, Stoytcheva M, Zlatev R, Gochev V (2012) Some clinical applications of the electrochemical biosensors. Mini-Rev Med Chem 12:1301-13013

4. Kröger S, Law RJ (2005) Sensing the sea. Trends Biotechnol 23:250-256

5. Viswanathan S, Radecka H, Radecki J (2009) Electrochemical biosensors for food analysis. Monatsh Chem 140:891-899

6. Telsnig D, Terzic A, Krenn T, Kassarnig V, Kalcher K, Ortner A (2012) Development of a voltammetric amine oxidase-modified biosensor for the determination of biogenic amines in food. Int $\mathrm{J}$ Electrochem Sci 7:6893-6903

7. Tichoniuk M, Gwiazdowska D, Ligaj M, Filipiak M (2010) Electrochemical detection of foodborne pathogen aeromonas hydrophila by DNA hybridization biosensor. Biosens Bioelectron 26:1618-1623

8. Luo C, Lei Y, Yan L, Yu T, Li Q, Zhang D, Ding S, Ju H (2012) A rapid and sensitive aptamer-based electrochemical biosensor for direct detection of Escherichia Coli O111. Electroanalysis 24:1186-1191

9. De Leo M, Kuhn A, Ugo P (2007) 3D-ensembles of gold nanowires: Preparation, characterization and electroanalytical peculiarities. Electroanalysis 19:227-236
10. Heim M, Reculusa S, Ravaine S, Kuhn A (2012) Engineering of complex macroporous materials through controlled electrodeposition in colloidal superstructures. Adv Funct Mater 22:538-545

11. Silvestrini M, Schiavuta P, Scopece P, Pecchielan G, Moretto LM, Ugo P (2011) Modification of nanoelectrode ensembles by thiols and disulfides to prevent non specific adsorption of proteins. Electrochim Acta 56:7718-7724

12. Pozzi Mucelli S, Zamuner M, Tormen M, Stanta G, Ugo P (2008) Nanoelectrode ensembles as recognition platform for electrochemical immunosensors. Biosens Bioelectron 23:1900-1903

13. Godino N, Borrise X, Munoz FX, del Campo FJ, Compton RG (2009) Mass transport to nanoelectrode arrays and limitations of the diffusion domain approach: Theory and experiment. J Phys Chem C 113:11119-11125

14. Zoski CG, Yang N, He P, Berdondini L, Koudelka-Hep M (2004) Addressable nanoelectrode membrane arrays: fabrication and steady-state behavior. Anal Chem 79:1474-1484

15. Arumugam PU, Chen H, Siddiqui S, Weinrich JAP, Jejelowo A, Li J, Meyyapan M (2009) Wafer-scale fabrication of patterned carbon nanofiber nanoelectrode arrays: a route for development of multiplexed, ultrasensitive disposable biosensors. Biosens Bioelectron 24:2818-2824

16. Martin CR (1999) In: Bard AJ, Rubinstein I (eds) Electroanalytical Chemistry. Marcel Dekker, New York

17. Menon VP, Martin CR (1995) Fabrication and evaluation of nanoelectrode ensembles. Anal Chem 67:1920-1928

18. Possin GE (1970) A method for forming very small diameter wires. Rev Sci Instrum 41:772-774

19. Williams WD, Giordano N (1984) Fabrication of $80 \AA$ metal wires. Rev Sci Instrum 55:410-412

20. Routkevitch D, Bigioni T, Moskovits M, Xu J-M (1996) Electrochemical fabrication of CdS nanowire arrays in porous anodic aluminum oxide template. J Phys Chem 100:14037-14047

21. Schoenberger C, van der Zande BMI, Fokkink LGJ, Henny M, Schmid C, Kruger M, Bachtold A, Huber R, Birk H, Staufer U (1997) Template synthesis of nanowires in porous polycarbonate membranes: electrochemistry and morphology. J Phys Chem B 101:5497-5505

22. Penner RM, Martin CR (1987) Preparation and electrochemical characterization of ultramicroelectrode ensembles. Anal Chem 59:2625-2630

23. De Leo M, Pereira FC, Moretto LM, Scopece P, Polizzi S, Ugo P (2007) Towards a better understanding of gold electroless deposition in track-etched templates. Chem Mater 19:5955-5964

24. Gilliam RJ, Thorpe SJ, Kirk DJW (2006) A nucleation and growth study of gold nanowires and nanotubes in polymeric membranes. Appl Electrochem 37:233-239

25. Gambirasi A, Cattarin S, Musiani M, Vázquez-Gómez L, Verlato E (2011) Direct electrodeposition of metal nanowires on electrode surface. Electrochim Acta 56:8582-8588

26. Konishi Y, Motoyama M, Matsushima H, Fukunaka Y, Ishii R, Ito $\mathrm{Y}$ (2003) Electrodeposition of $\mathrm{Cu}$ nanowire arrays with a template. J Electroanal Chem 559:149-153

27. Motoyama M, Fukunaka Y, Sakka T, Ogata YH, Kikuchi S (2005) Electrochemical processing of $\mathrm{Cu}$ and $\mathrm{Ni}$ nanowire arrays. J Electroanal Chem 584:84-91

28. Piraux L, Duboix S, Champagne S (1997) Template synthesis of nanoscale materials using the membrane porosity. Nucl Inst Methods Phys Res B 131:357-363

29. Chiriac H, Moga AE, Urse M, Ovari T-A (2003) Preparation and magnetic properties of electrodeposited magnetic nanowires. Sensors Actuators A 106:348-351

30. Pirota KR, Navas D, Hernandez-Vélez M, Nielsch K, Vasquez M (2004) Novel magnetic materials prepared by electrodeposition techniques: arrays of nanowires and multi-layered microwires. J Alloy Compd 369:18-26 
31. Platt M, Dryfeand RAW, Robaerts EPL (2004) Structural and electrochemical characterisation of Pt and Pd nanoparticles electrodeposited at the liquid/liquid interface. Electrochim Acta 49:39373945

32. Prieto AL, Sander MS, Gonzalez MSM, Gronsky R, Sands T, Stacy AM (2001) Electrodeposition of ordered $\mathrm{Bi}_{2} \mathrm{Te}_{3}$ nanowire arrays. J Am Chem Soc 123:7160-7161

33. Paunovic M, Schlesinger M (2000) Modern electroplating. Modern Electroplating. Wiley, New York

34. Pereira FC, Moretto LM, De Leo M, Boldrin Zanoni MV, Ugo P (2006) Electrochemistry of phenothiazine and methylviologen biosensor electron-transfer mediators at nanoelectrode ensembles. Anal Chim Acta 575:16-24

35. Jirage KB, Hulteen JC, Martin CR (1997) Nanotubule-based molecular-filtration membranes. Science 278:655-658

36. Hulteen JC, Jirage KB, Martin CR (1998) Introducing chemical transport selectivity into gold nanotubule membranes. J Am Chem Soc 120:6603-6604

37. Jirage KB, Hulteen JC, Martin CR (1999) Effects of thiol chemisorption on the transport properties of gold nanotubule membranes. Anal Chem 71:4913-4918

38. Kobayashi Y, Martin CR (1999) Highly-sensitive methods for electroanalytical chemistry based on nanotubule membranes. Anal Chem 71:3665-3672

39. Bercu B, Enculescu I, Spohr R (2004) Copper tubes prepared by electroless deposition in ion track templates. Nucl Inst Methods B 225:497-502

40. Dryfe RAW, Simm AO, Kralj B (2003) Electroless deposition of palladium at bare and templated liquid/liquid interfaces. J Am Chem Soc 125:13014-13015

41. Tai Y-L, Teng H (2004) Template synthesis and electrochemical characterization of Nickelbased tubule electrode arrays. Chem Mater 16:338-342

42. Krishnamoorthy K, Zoski CG (2005) Fabrication of 3D gold nanoelectrode ensembles by chemical etching. Anal Chem 77:5068-5071

43. Yu S, Li N, Wharton J, Martin CR (2003) Nano wheat fields prepared by plasma-etching gold nanowire-containing membranes. Nano Lett 3:815-818

44. Ugo P, Moretto LM, Vezzà F (2002) Ionomer-coated electrodes and nanoelectrode ensembles as electrochemical environmental sensors: Recent advances and prospects. ChemPhysChem 3:917-925

45. Ugo P (2005) In: Grimes CA, Dickey EC, Pishko MV (eds) Encyclopedia of sensors. American Scientific Publishers, Stevenson Ranch

46. Ugo P, Moretto LM (2007) In: Zoski C (ed) Handbook of Electrochemistry. Amsterdam, Elsevier

47. Moretto LM, Pepe N, Ugo P (2004) Voltammetry of redox analytes at trace concentrations with nanoelectrodes ensembles. Talanta 62:1055-1060

48. Arrigan DWM (2004) Nanoelectrods, Nanoelectrode arrays and their application. Analyst 129:1157-1165

49. Errachid A, Mills CA, Pla-Roca M, Lopez MJ, Villanueva G, Bausells J, Crespo E, Teixidor F, Samitier J (2008) Focused ion beam production of nanoelectrode arrays. Mater Sci Eng C 28:777-780

50. Lanyon YH, De Marzi G, Watson YE, Quinn AJ, Gleeson JP, Redmond G, Arrigan DWM (2007) Fabrication of nanopore array electrodes by focused ion beam milling. Anal Chem 79:3048-3055

51. Sandison ME, Cooper JM (2006) Nanofabrication of electrode arrays by electron-beam and nanoimprint lithographies. Lab Chip 6:1020-1025

52. Losilia NS, Martinez J, Garcia R (2009) Large area nanoscale patterning of silicon surfaces by parallel local oxidation. Nanotechnology 20:475304

53. Losilia NS, Oxtoby NS, Martinez J, Garcia F, Garcia R, Mas-Torrent M, Vecciana J, Rovia C (2008) Sub-50 nm positioning of organic compounds onto silicon oxide patterns fabricated by local oxidation nanolithography. Nanotechnology 19:455308

54. Albonetti C, Martinez J, Losilia NS, Greco P, Cavallini M, Borgatti F, Montecchi M, Pasquali L, Garcia R, Biscarini F (2008) Parallellocal anodic oxidation of silicon surfaces by soft stamps. Nanotechnology 19:435303

55. Moretto LM, Tormen M, De Leo M, Carpentiero A, Ugo P (2011) Polycarbonate-based ordered arrays of electrochemical nanoelectrodes obtained by e-beam lithography. Nanotechnology 22:185305

56. Zamuner M, Pozzi Mucelli S, Tormen M, Stanta G, Ugo P (2008) Electrochemical nanobiosensors and protein detection. Eur $\mathrm{J}$ Nanomed 1:33-36

57. Bard AJ, Faulkner L (2000) Electrochemical Methods. VCH, Weinheim, ch. 5

58. Dickinson EJF, Compton RG (2009) Diffuse double layer at nanoelectrodes. Phys Chem Lett C 113:17585-17589

59. Henstridge MC, Compton RG (2011) Mass transport to micro- and nanoelectrodes and their arrays: a review. Chem Rec 12:63-71

60. Lee HJ, Beriet C, Ferrigno R, Girault HH (2001) Cyclic voltammetry at a regular microdisc electrode array. J Electroanal Chem 502:138-145

61. Hulteen JC, Menon VP, Martin CR (1996) Template preparation of nanoelectrode ensembles achieving the 'pure-radial' electrochemicalresponse limiting case. J Chem Soc Faraday Trans 92:4029-4032

62. Cheng JF, Whitley LD, Martin CR (1989) Ultramicroelectrode ensembles. Comparison of experimental and theoretical responses and evaluation of electroanalytical detection limits. Anal Chem 61:762-766

63. Guo J, Lindner E (2009) Cyclic voltammograms at coplanar and shallow recessed microdisk electrode arrays: guidelines for design and experiment. Anal Chem 81:130-138

64. Davies TJ, Compton RG (2005) The cyclic and linear sweep voltammetry of regular and random arrays of microdisc electrodes: Theory. J Electroanal Chem 585:63-82

65. Huang X-J, O’Mahony AM, Compton RG (2009) Microelectrode arrays for electrochemistry: Approaches to fabrication. Small 7:776 788

66. Amatore C, Oleinik AI, Svir I (2009) Numerical simulation of diffusion processes at recessed disk microelectrode arrays using the quasi-conformal mapping approach. Anal Chem 81:4397-4405

67. Ugo P, Moretto LM, De Leo M, Doherty AP, Vallese C, Pentlavalli $S$ (2010) Diffusion regimes at nanoelectrode ensembles in different ionic liquids. Electrochim Acta 55:2865-2872

68. Ugo P, Moretto LM, Vezzà F (2003) In: Baltes H, Fedder GK, Korvink JG (eds) Sensors Update. Wiley-VCH, Weinheim

69. Ugo P, Moretto LM, Bellomi S, Menon VP, Martin CR (1996) Ion exchange voltammetry at polymer film coated nanoelectrode ensembles. Anal Chem 68:4160-4165

70. Brunetti B, Ugo P, Moretto LM, Martin CR (2000) Electrochemistry of phenothiazine and methylviologen biosensor electrontransfer mediators at nanoelectrode ensembles. J Electroanal Chem 491:166-174

71. Amatore C, Saveant JM, Tessier D (1983) Charge transfer at partially blocked surfaces. A model for the case of microscopic active and inactive sites. J Electroanal Chem 147:39-51

72. Greef R, Pea R, Peter LM, Pletcher D, Robinson J (1985) Instrumental Methods in Electrochemistry. Ellis Horwood Ltd., Chester

73. Nicholson RS (1965) Theory and application of cyclic voltammetry for measurement of electrode reaction kinetics. Anal Chem 37:1351-1355

74. Triroj N, Jaroenapibal P, Shi H, Yeh JI, Beresford R (2011) Microfluidic chip-based nanoelectrode array as miniaturized biochemical sensing platform for prostate-specific antigen detection. Biosens Bioelectron 26:2927-2933

75. Ugo P, Pepe N, Moretto LM, Battagliarin M (2003) Voltammetry in the presence of ultrasound: sonovoltammetric detection of 
cytochrome $c$ under very fast mass transport conditions. J Electroanal Chem 560:51-58

76. Hill HA, Nakagawa Y, Marken F, Compton RG (1996) Direct voltammetry of cytochrome $c$ at trace concentrations with nanoelectrode ensembles. J Phys Chem 100:17395-17399

77. Eddowes MJ, Hill HAO (1977) Novel method for investigation of electrochemistry of metalloproteins - cytochrome- $c$. J Chem Soc Chem Commun 771-772

78. Allen PM, Hill HAO, Walton NJ (1984) Surface modifiers for the promotion of direct electrochemistry of cytochrome. J Electroanal Chem 178:69-86

79. Eddowes JM, Hill HAO (1979) Electrochemistry of horse heart cytochrome $c$. J Am Chem Soc 101:4461-4462

80. Sagara T, Murakami H, Igarashi S, Sato H, Niki K (1991) Redox mechanism of cytochrome $c$ at modified gold electrodes. Langmuir 7:3190-3196

81. Sagara T, Niwa K, Sone A, Innen C, Niki K (1990) Redox reaction mechanism of cytochrome $c$ at modified gold electrodes. Langmuir 6:254-262

82. Lin Y, Lu F, Tu Y, Ren Z (2004) Glucose biosensors based on carbon nanotube nanoelectrode ensembles. Nano Lett 4:191-195

83. Cao L, Yan P, Sun K, Kirk DW (2008) Tailor-made gold brush nanoelectrode ensembles modified with 1-cysteine for the detection of daunorubicine. Electrochim Acta 53:81448148

84. Cao L, Yan P, Sun K, Kirk DW (2009) Gold 3D brush nanoelectrode ensembles with enlarged active area for the direct voltammetry of daunorubicin. Electroanalysis 21:1183-1188

85. Gasparac R, Taft BJ, Lapierre-Devlin MA, Lazareck AD, Xu JM, Kelley SO (2004) Ultrasensitive electrocatalytic DNA detection at two- and three-dimensional nanoelectrodes. J Am Chem Soc 126:12270-12271

86. Lapierre-Devlin MA, Asher CL, Taft BJ, Gasparac R, Roberts MA, Kelley SO (2005) Amplified electrocatalysis at DNA-modified nanowires. Nano Lett 5:1051-1055

87. Silvestrini M, Fruk L, Ugo P (2012) Functionalized ensembles of nanoelectrodes as affinity biosensors for DNA hybridization detection. 40:265-270

88. Silvestrini M, Ugo P (2012) Ensembles of nanoelectrodes modified with gold nanoparticles: Characterization and application to DNAhybridization detection. Anal Bioanal Chem doi: 10.1016/ j.bios.2012.07.041

89. Fruk L, Müller J, Weber G, Narvaez A, Dominguez E, Niemeyer CM (2007) DNA-directed immobilization of horseradish peroxidase-DNA conjugates on microelectrode arrays: Towards electrochemical screening of enzyme libraries. Chem Eur 13:5223-5231 